REVISTA DE INVESTIGACIÓN

EN GESTIÓN CULTURAL

Córima, Revista de Investigación en Gestión Cultural

ISSN electrónico: 2448-7694

Universidad de Guadalajara

Sistema de Universidad Virtual

México

corima@udgvirtual.udg.mx

Año 6, número 11, julio-diciembre 2021

\title{
Mapeo de los observatorios culturales en Iberoamérica: revisión desde la gestión cultural
}

\section{Mapping of cultural observatories in Ibero-America: review from cultural management}

\author{
José Luis Mariscal Orozco ${ }^{1}$ \\ Melissa Zanini Anguiano 2 \\ Universidad de Guadalajara, México
}

https://doi.org/10.32870/cor.a6n11.7408

[Recibido: 30/4/2021; aceptado para su publicación: 18/5/2021]

\section{Resumen}

Este artículo presenta los resultados de una investigación que tiene como objetivo mapear los observatorios culturales con vías a la identificación de la producción y la gestión de conocimiento especializado en la gestión cultural. Para esto, se diseñó e implementó un estudio de tipo exploratorio descriptivo, cuyo método mixto recoge y analiza datos

\footnotetext{
1 Antropólogo y gestor cultural. Profesor investigador en el Sistema de Universidad Virtual, Universidad de Guadalajara, México. Miembro del Sistema Nacional de Investigadores. Doctor y maestro en Antropología Social (Colegio de Michoacán, México). Cuenta con perfil PRODEP. Correo electrónico: mariscal@udgvirtual.udg.mx. ORCID: http://orcid.org/0000-0001-6769-0761

2 Correo electrónico: zaninimeel1@gmail.com

CÓMO CITAR ESTE ARTÍCULO

Mariscal Orozco, J. L. y Zanini Anguiano, M. (2021). Mapeo de los observatorios culturales en Iberoamérica: revisión desde la gestión cultural. Córima, Revista de Investigación en Gestión Cultural, 6(11). DOI: 10.32870/cor.a6n11.7408
} 
cuantitativos y cualitativos sobre la identificación y la caracterización de los observatorios culturales en Iberoamérica a través de la revisión documental. A partir de la información analizada, se identifica una tendencia a centrar la observación en las políticas culturales y la economía de la cultura, esto debido a que más de la mitad de los observatorios son promovidos por instituciones gubernamentales y universitarias. De la misma manera, se observa que hay un predominio por la conformación de sistemas de información y de la divulgación de actividades, pues se privilegia la generación de diagnósticos y repositorios de información para la toma de decisiones, y son escasos los modelos de trabajo basados en la gestión del conocimiento, tendencia actual que se encuentra en otros observatorios sociales basados en la ciencia abierta y colaborativa.

\section{Palabras clave}

Gestión cultural; formalización disciplinar; mapeos culturales; observatorios culturales; indicadores culturales.

\section{Abstract}

This article presents the results of a research that aims to map cultural observatories with ways to identify the production and management of specialized knowledge in cultural management. For this, a descriptive exploratory study was designed and implemented, whose mixed method collects and analyzes quantitative and qualitative data on the identification and characterization of cultural observatories in Ibero-America through documentary review. From the analyzed information, a tendency to focus the observation on cultural policies and the economy of culture is identified, because more than half of the observatories are promoted by government and university institutions. In the same way, it is observed that there is a predominance due to the formation of information systems and the dissemination of activities, since the generation of diagnoses and information repositories for decision-making is privileged, with few work models based on management knowledge, a current trend found in other social observatories that are based on open and collaborative science.

\section{Keywords}

Cultural management; disciplinary formalization; cultural mapping; cultural observatories; cultural indicators.

\section{Introducción}

En las últimas tres décadas, hemos sido testigos de un intenso proceso de formalización de la gestión cultural en la región iberoamericana, que comenzó a 
partir del reconocimiento de prácticas relacionadas con el diseño y la operación de la acción cultural, la demanda de mano de obra especializada en las nuevas instituciones culturales de los estados democráticos y la responsabilidad asumida por los Estados nación de hacerse cargo de diseñar e implementar lineamientos y políticas públicas enfocadas al ámbito cultural (Martinell, 2009), pero también por la necesidad de contar con nuevos instrumentos que permitieran rentabilizar las iniciativas artísticas y promover la generación de nuevos mercados de consumo cultural (Bayardo, 2018).

Como parte de este proceso de formalización, diversas instituciones gubernamentales y universitarias implementaron acciones para profesionalizar a los gestores culturales que aprendieron el oficio desde la práctica a través de dos grandes estrategias: a) la capacitación del personal de las instituciones culturales, así como de algunos agentes comunitarios a través de cursos, seminarios y diplomados; y b) el surgimiento de programas de formación profesional a nivel superior, tanto de licenciatura como de posgrado.

La primera estrategia se implementó debido a la participación activa de las instituciones gubernamentales y educativas de los países iberoamericanos, así como de organismos internacionales como la Organización de Estados Iberoamericanos, la Agencia Española de Cooperación Internacional para el Desarrollo (AECID) y la Organización de las Naciones Unidas para la Educación, la Ciencia y la Cultura (UNESCO). Estas acciones de capacitación sirvieron para diseñar planes de estudio a nivel universitario, cuyos perfiles de egreso, competencias y ejes de formación estaban relacionados con los perfiles laborales existentes en ese momento (Mariscal, 2006; Rubim, Barbalho y Costa, 2012; Brambila, 2015).

Durante la primera década del siglo XXI, se registró un crecimiento exponencial de las ofertas académicas especializadas en gestión cultural, tanto de licenciatura como de maestría. En la mayoría de los casos, los cuerpos docentes estaban compuestos por gestores culturales que habían aprendido en la práctica y por académicos de otros campos disciplinares y profesionales que realizaron aportes significativos desde su formación y experiencia (Zapata, 2004; Arreola, Brambila y Mariscal, 2012; González, 2018).

En la medida en que los programas universitarios maduran, las universidades se han visto en la necesidad de consolidar sus núcleos académicos 
con profesores de tiempo completo que, además de la docencia, tienen como funciones sustanciales la investigación, el trabajo colegiado y la difusión científica. Paralelo a esto, en diversos congresos, encuentros y debates académicos, tanto nacionales como internacionales, se comenzó a visualizar que la gestión cultural no solo se estaba consolidando como una nueva profesión, sino también como un campo académico emergente en plena construcción y delimitación, en relación con otros campos disciplinarios.

Uno de los elementos clave en la construcción de la matriz disciplinaria de todo campo académico son las aportaciones teóricas que se dan desde una generalización de enfoques y valores compartidos por la comunidad académica emergente. Lo anterior se puede observar a partir de las investigaciones que se realizan y sus productos derivados de ellas.

No obstante, y debido a lo reciente que es este fenómeno, la información relacionada con la investigación en gestión cultural es escasa, y los resultados de los estudios en curso aún son preliminares, por lo que es importante realizar indagaciones exploratorias que permitan comprender el proceso de construcción disciplinar de la gestión cultural. Así, como parte de los trabajos de investigación ${ }^{3}$ que realiza el Observatorio Latinoamericano de Gestión Cultural, se pretende realizar un mapeo de la oferta académica actual en gestión cultural a nivel superior, así como del desarrollo de su producción académica científica y humanística.

Este artículo presenta los resultados de una investigación exploratoria que pretende hacer un mapeo de los observatorios culturales en Iberoamérica desde la mirada de la gestión cultural. Los observatorios tienen un papel importante en la generación y gestión de conocimiento especializado, no solo para la toma de decisiones en términos de políticas públicas, sino también en la producción de nuevo conocimiento que sirve, a su vez, para la reproducción de la práctica profesional a través de la actualización de los planes y programas de estudio.

\footnotetext{
${ }^{3}$ Este documento muestra algunos resultados preliminares del proyecto "Mapeo de la producción y reproducción del conocimiento académico de la gestión cultural en Latinoamérica" del Observatorio Latinoamericano de Gestión Cultural.
} 


\section{Marco teórico}

A través de la historia y en diferentes culturas, ha existido la figura de los observatorios como espacios donde se pueden contemplar los fenómenos naturales, principalmente los astronómicos. La idea del observatorio como un lugar para la visualización, el monitoreo y el análisis de los fenómenos, es aplicada al campo social en 1962, lo que enfatizó las políticas urbanas como objeto de estudio (Angulo, 2009).

La definición de observatorio en las ciencias sociales ha cambiado a lo largo del tiempo, en concordancia con las funciones que se le han asignado. Una de las primeras acciones fue la visualización y el registro de algún fenómeno social, temático u objeto de investigación, con el fin de dar seguimiento a su desarrollo:

Un observatorio es un organismo creado por un colectivo con el fin de seguir la evolución de un fenómeno de interés general, desde las administraciones públicas, nacionales, regionales y locales, desde instituciones académicas, sindicatos, empresas, fundaciones y organizaciones de la sociedad civil, se ha promovido y apoyado la creación de estos órganos coadyuvantes al desempeño institucional mediante señalamientos puntuales y propositivos previo al estudio, registro y análisis de la situación y evolución de una temática determinada (Instituto Nacional de Administración Pública, 2014, p. 15).

A esta función se le sumaron otras más, como definir los indicadores para la medición del fenómeno observado, así como los instrumentos de recuperación de la información, construidos de tal manera que permitan transformar los datos estadísticos en datos útiles para la toma de decisiones (Gregorio en Angulo, 2009). Aquellos observatorios relacionados con las políticas públicas suelen tener como objetivos:

Proveer información útil, oportuna y fiable, relacionada con el quehacer o temas relacionados con la administración pública, que es utilizada por los hacedores o gestores públicos en sus tareas administrativas al frente de las entidades públicas.

Proveer información sobre la marcha institucional, ya sea de sus políticas, programas y proyectos, permitiendo su análisis, evaluación, monitoreo y vigilancia por los propios servidores públicos, por los ciudadanos o por los distintos grupos de interés que interactúan con la administración pública (Chevarria, Inga, De Pasquale y Holmberg, 2015, p. 10). 
Otra función clave que se le ha asignado a los observatorios sociales es la socialización y transferencia del conocimiento generado, ya sea a través de publicaciones, informes y conferencias, o bien, a través de la capacitación y formación de agentes involucrados en la toma de decisiones y en el desarrollo de nuevos equipos de investigación que permitan la continuidad y la mejora del trabajo investigativo. Es a través de sus sitios web donde:

se reúnen un conjunto de aplicaciones e información dispuestas de forma central donde no solo se consulta el material, sino el 'estado del arte', comportamientos, corrientes y tendencias sobre la investigación y desarrollo del conocimiento sobre algún tema, y por tanto, permiten la interacción en un público tan abierto que lo mismo sirve al observador ocasional como al analista o al experto, pues el valor agregado del observatorio se sustenta en: 1) buscar la información, 2) discernir su relevancia, 3) organizarla de modo coherente y 4) presentarla de forma clara (Gobierno Vasco, en Angulo, 2009, p. 6).

Con el desarrollo de las tecnologías de información y comunicación fue posible la recuperación, el registro, la sistematización y el análisis de datos de manera descentralizada, además de que permitió no solo documentar datos de fuentes análogas, sino también de digitales, lo cual posibilitó la utilización de herramientas de gestión de la información (Sancho, 2004). Esto creó las condiciones para que el trabajo de los observatorios se desconcentrara y permitiera la participación de diversas personas y grupos ubicados en distintas localidades, los cuales aportaron y compartieron datos que pudieron ser contrastados e integrados en los procesos de análisis e interpretación.

En este sentido, las funciones asignadas a los observatorios sociales convergen en un modelo cada vez más recurrente, que está relacionado con la generación de sistemas de gestión de la información y del conocimiento, con lo que se concibe así al observatorio como "la gran estrategia de gestión del conocimiento que se vale de todas sus herramientas para transformar datos en información e información en conocimiento, además de incentivar la conversión del conocimiento tácito en conocimiento explícito" (Angulo, 2009, p. 12), y se consideran para esto "herramientas y conceptos propios de la gestión del conocimiento, como es el caso de las buenas prácticas, lecciones aprendidas, directorio de saberes, vigilancia estratégica, redes de colaboración y uso compartido del conocimiento" (Angulo, 2009, p. 12). 
Los observatorios culturales surgen en la década de los noventa como una forma de documentar los recursos culturales existentes, pero también para sistematizar y articular diferentes fuentes de información relacionadas con el sector cultural (Ortega, 2010). Son un tipo de observatorio social, pero enfocado en el ámbito cultural: "los observatorios culturales son organismos responsables de facilitar el acceso a la información y de su conversión en conocimiento cultural para ayudar en la toma de decisiones mediante un sistema de información sistemático" (Ortega, Claudio y Bunten, 2011, pp. 14-15). Lo anterior implica un énfasis primordial en la aplicación de la información generada en la agenda de las políticas públicas del sector cultural (Piedras, 2008).

De acuerdo con Ben (2014), los observatorios culturales realizan diversas actividades o productos que permiten hacer tangible y estratégica la información que se genera en los procesos de investigación con prácticas concretas en el sector cultural:

1) Generación de información estadística, organizada y sistematizada, predispuesta de tal manera que permita priorizar problemáticas, áreas de oportunidad y desarrollo de estrategias en el diseño e implementación de políticas públicas.

2) Conformación de sistemas de información (con énfasis en lo cualitativo) que organicen y sistematicen una gran diversidad de indicadores regularmente territorializados.

3) Construcción de cartografías culturales que permitan mapear y visualizar los recursos culturales en los territorios organizados por categorías analíticas de utilidad para los planificadores territoriales.

4) Realización de proyectos $I+D+i$ que permitan la recolección, la sistematización, el análisis y la interpretación de los datos desde una perspectiva de resolución de problemas a partir de la innovación de la acción cultural.

5) Brindar recomendaciones a instancias gubernamentales e iniciativas privadas y civiles a partir de la observación de tendencias y casos de éxito (Ben, 2014).

Existen diversas formas institucionales y alcances de los observatorios culturales, de acuerdo con las necesidades y contextos desde donde surgen las 
iniciativas de su conformación; algunos pertenecen a instituciones gubernamentales, otros a universidades, otros a ciudadanos y algunos más son mixtos, con alcances locales, nacionales e internacionales. Además, existen interrelaciones con otros campos disciplinares (como antropología y urbanismo), pero también intersectoriales (como economía y política pública), por lo que algunos observatorios pueden no ser considerados en la categoría de "culturales" como tal, pero generan información relevante para el sector cultural.

Ante esta diversidad de funciones, formas institucionales y alcances, toma relevancia la identificación de los observatorios, especialmente de las iniciativas que permitan caracterizarlos y documentar sus acciones, alcances y resultados en el sector cultural. Por lo anterior, se planteó la realización de un mapeo de los observatorios culturales en Iberoamérica para comprender su estado actual.

No obstante, este ejercicio de mapeo se ha realizado en otras investigaciones. Entre los estudios más relevantes ${ }^{4}$ se encuentran los de Puente (2016), Martínez, Garrido y Lugo (2019) y Sarmiento, Delgado e Infante (2019), para el caso de los observatorios sociales. En el caso de los observatorios culturales, se encuentran importantes investigaciones de Vázquez (2011), Abeledo (2014) y Giovinazzo (2014), quienes realizaron un ejercicio de tipología y caracterización de sus funciones e impactos. De igual manera, los trabajos preliminares de mapeo y caracterización de los observatorios culturales en Iberoamérica de Negrón y Brodsky (2014) y de Mariscal y Rucker (2016) permiten un acercamiento a su especificación en términos de sus formas institucionales y temáticas abordadas.

Las investigaciones de Ortega (2010), Ortega y del Valle (2010) y Ortega, Claudio y Bunten (2011), a pesar de que están enfocadas en la comunidad europea, tienen una gran importancia metodológica, ya que articulan tres grandes categorías que otros estudios mencionados analizan de forma separada: 1) la identificación del observatorio cultural en términos de ubicación geográfica y su descripción; 2) la caracterización institucional, temática y de propósitos, y 3) las formas y alcances del trabajo de investigación.

Así, se conceptualiza el observatorio cultural como un espacio (físico o virtual) donde se recupera, documenta, organiza, sistematiza, analiza e interpreta

\footnotetext{
${ }^{4}$ Se trata de estudios relevantes para nuestro proyecto, ya sea por los resultados que comparten la metodología utilizada o por las temáticas analizadas y sus fuentes consultadas.
} 
información relacionada con el sector cultural para la generación, socialización y transferencia del conocimiento presentado, de tal manera que permita la comprensión del estado actual y la toma de decisiones para el diseño y la operación de la acción cultural (en cualquiera de sus niveles y formas).

Se parte de este amplio concepto para dar cabida a las diferentes iniciativas surgidas en Iberoamérica que se autodenominan como "observatorio cultural". Desde una mirada académica ortodoxa, tal vez muchas de estas iniciativas no pueden ser clasificadas como observatorios; sin embargo, consideramos que estas instancias de investigación se encuentran en plena construcción, pues se relacionan con los procesos de institucionalización de la gestión cultural en la región, por lo que, en algunos casos, se complejizan y oficializan, y en algunos otros, desaparecen al no tener continuidad por falta de un soporte financiero, institucional o de equipo de trabajo. En cualquiera de los casos anteriores, se trata de iniciativas valiosas para su documentación como parte de la observación de la formalización disciplinar.

\section{Metodología}

El proyecto "Mapeo de la producción y reproducción del conocimiento académico de la Gestión Cultural en Latinoamérica" tiene como objetivo general analizar el estado actual de los programas de formación y de los grupos de investigación como parte del proceso de institucionalización académica de la gestión cultural como campo disciplinar en Latinoamérica. Uno de sus objetivos particulares es mapear los observatorios culturales con vías a la identificación de la producción y gestión de conocimiento especializado en gestión cultural. ${ }^{5}$

Para el logro de este objetivo específico, se diseñó e implementó un estudio de tipo exploratorio descriptivo, cuyo método mixto recoge y analiza datos cuantitativos y cualitativos. Se tomó como referencia el estudio realizado por Ortega, Claudio y Bunten (2011), por lo que se tomaron algunos indicadores pertinentes y se realizaron algunos ajustes de acuerdo con los intereses y los

\footnotetext{
5 Si bien es cierto que el proyecto general hace énfasis en lo latinoamericano, el mapeo de los observatorios se consideró también a España y Portugal, esto debido a su importancia e incidencia en la investigación que se hace en Latinoamérica.
} 
objetivos de la investigación. Así, se definieron tres categorías de análisis con sus respectivas variables, las cuales se enuncian a continuación.

\section{Identificación}

Contiene indicadores básicos de identificación del observatorio analizado, como nombre, país, ubicación, sitio web y descripción general, brindada por el mismo observatorio.

\section{Caracterización}

Recaba información que permita caracterizar institucional y disciplinariamente al observatorio. Para esto, se consideraron las instituciones que promueven el surgimiento y el mantenimiento del observatorio, las cuales pueden ser:

a) Organismos internacionales, como la Organización de Estados Iberoamericanos (OEI) o la Organización de las Naciones Unidas para la Educación, la Ciencia y la Cultura (UNESCO).

b) Instituciones gubernamentales, como ministerios de cultura o secretarías de educación o turismo.

c) Instituciones, como universidades o centros de investigación.

d) Sociedad civil, como asociaciones civiles o fundaciones.

e) Mixto, en el que participa más de una categoría mencionada.

Otros elementos a considerar fueron los objetivos o propósitos y las líneas de investigación que el observatorio definía en sus documentos oficiales o sitios web. De la misma manera, se consideró el año de surgimiento para poder identificar alguna tendencia en la aparición de los observatorios y su permanencia en el tiempo.

Asimismo, se consideraron sus temáticas de interés, las cuales se retomaron del estudio de Mariscal y Rucker (2016) debido a que sirven como categorías analíticas para agrupar diferentes estudios que se realizan desde otras disciplinas o sectores, pero que son de interés para la gestión cultural. Estas temáticas son: 
1) Políticas culturales: diseño e impacto de las políticas culturales en diversos niveles de observación.

2) Economía de la cultura: información sobre la relación entre la economía y el ámbito cultural, así como los datos estadísticos de los mismos.

3) Derecho cultural: recopilación y análisis de los datos referentes al derecho humano, a la cultura y a sus procesos de institucionalización.

4) Educación: información sobre procesos, experiencias y políticas educativas, y su incidencia en el sector cultural.

5) Patrimonio cultural: generación y socialización de información relevante sobre los patrimonios culturales tangibles e intangibles.

6) Fenómenos culturales: observación de prácticas, servicios, bienes y problemáticas del sector cultural.

7) Turismo cultural: análisis de los fenómenos vinculados al turismo cultural.

8) Cooperación cultural: recolección de información relacionada con la cooperación cultural y la promoción del desarrollo territorial.

9) Cultura y comunicación: análisis de la configuración y el impacto de la comunicación de la cultura. ${ }^{6}$

10) Extensión cultural: estudio de los modelos, el impacto y las actividades de extensión y difusión cultural de las universidades.

11) Actividades culturales: documentación, análisis y difusión de las actividades culturales que se realizan en ciertos territorios o con algún tema de interés.

Finalmente, se les asignó una palabra clave, las cuales han sido definidas por el Observatorio Latinoamericano de Gestión Cultural y forman parte de una lista de términos normalizados en su sistema de gestión de la información. Este vocabulario controlado se ha definido desde criterios disciplinares de la gestión cultural como una forma de organización del conocimiento generado y utilizado en este campo disciplinar. ${ }^{7}$

\footnotetext{
${ }^{6}$ Para este estudio no se consideraron los observatorios de comunicación que centran su análisis en los medios de comunicación (los cuales son abundantes y variados en sus formas organizacionales), ya que estos tienen intereses de investigación distintos a la gestión cultural.

7 Para más información al respecto, consultar: https://observatoriocultural.udgvirtual.udg.mx/estructura-y-organizacion
} 


\section{Formas y alcances del trabajo}

A esta categoría corresponden variables que permiten comprender cómo se organiza el trabajo del observatorio y las acciones que realizan. Por lo anterior, se consideró el tipo de actividades que llevan a cabo como parte de las funciones que establecen y el alcance territorial de sus investigaciones. Asimismo, se identificaron sus estudios relacionados con la gestión cultural y se consideraron las siguientes opciones:

a) La dedicación total a las investigaciones sobre gestión cultural.

b) La gestión cultural solo es uno de los intereses de la investigación.

c) La realización de algunos trabajos relacionados con la gestión cultural.

En relación con las formas de trabajo, se tomaron los siguientes modelos propuestos por Mariscal y Rucker:

a) Sistemas de información cultural: recuperan, sistematizan y organizan la información sobre indicadores culturales estandarizados y almacenados en bases de datos, dispuestos para su consulta en línea, o bien, a través de boletines o informes con resúmenes o diagnósticos temáticos.

b) Grupo de expertos: convocan o invitan a expertos (regularmente, académicos) para analizar y debatir sobre un tema actual y de interés para el observatorio. De esa manera, se generan diagnósticos, estados del arte o posicionamientos que aprovechan la trayectoria y el trabajo previo de sus participantes.

c) Grupos de investigación: participan uno o más grupos de personas que comparten proyectos de investigación orientados a las líneas de investigación del observatorio. Los resultados se socializan como producción académica o de divulgación en encuentros académicos o en su sitio web. 
d) Sistemas de referencias: identifican, recuperan, documentan y socializan recursos informativos académicos o noticiosos relevantes a sus temas de interés.

e) Gestión del conocimiento: recuperan, documentan, sistematizan y organizan datos para la conformación de sistemas de información a través de proyectos de investigación, bajo un modelo de ciencia abierta y colaborativa y con el uso de herramientas digitales que les permitan la gestión del conocimiento generado por los grupos de investigación.

Tabla 1. Resumen de categorías y variables de análisis

\begin{tabular}{|l|l|}
\hline \multicolumn{1}{|c|}{ Categoría } & \multicolumn{1}{c|}{ Variables } \\
\hline Identificación & $\begin{array}{l}\text { - Nombre } \\
\text { - Ciudad } \\
\text { - País } \\
\text { - Ubicación } \\
\text { - Sitio web } \\
\text { - Descripción general }\end{array}$ \\
\hline Caracterización & $\begin{array}{l}\text { - Institución promotora } \\
\text { - Objetivo o propósito } \\
\text { - Temáticas } \\
\text { - Líneas de investigación } \\
\text { - Año de creación } \\
\text { - Palabras clave }\end{array}$ \\
\hline Formas y alcances del trabajo & $\begin{array}{l}\text { - Modelo de trabajo del } \\
\text { observatorio }\end{array}$ \\
& $\begin{array}{l}\text { - Actividades principales que } \\
\text { realiza } \\
\text { - Dedicación a la gestión cultural } \\
\text { - Alcance de sus investigaciones }\end{array}$ \\
\hline
\end{tabular}

Fuente: elaboración propia.

Al tratarse de un estudio exploratorio, se optó por una muestra no estadística que utilizó la técnica de bola de nieve. Los criterios de selección de los observatorios fueron:

1) Que cuente con un sitio web y con información básica (al menos el $70 \%$ de los indicadores).

2) Que se autodefina como observatorio. 
3) Que sea promovido o sostenido por una instancia con sustento organizacional, ya sea gubernamental, universitaria, privada o civil (no se consideran iniciativas de tipo personal).

4) Que realice actividades o tenga algún producto resultado de la organización, la sistematización o la gestión de la información.

5) Que las temáticas de sus acciones de investigación, documentación o investigación pertenezcan explícitamente al ámbito cultural y, preferentemente, que estén relacionadas con la acción cultural.

A partir de estos criterios de selección, se delimitaron los siguientes sujetos de estudio (ver tabla 2).

Tabla 2. Relación de observatorios considerados para el estudio

\begin{tabular}{|l|l|l|}
\hline Núm. & \multicolumn{1}{|c|}{ Observatorio } & \multicolumn{1}{|c|}{ País } \\
\hline & Observatorio del Patrimonio Histórico Cultural & Argentina \\
\hline & Observa Cultura UBA & Argentina \\
\hline & Observatorio Cultural de Buenos Aires & Argentina \\
\hline & Observatorio de Industrias Culturales de la Ciudad de Buenos Aires & Argentina \\
\hline & Observatorio de Industrias Creativas & Argentina \\
\hline & Sistema de Información Cultural de la Argentina (SInCA) & Argentina \\
\hline & Observatório de Museus e Centros Culturais & Brasil \\
\hline & Observatório da cultura & Brasil \\
\hline & Observatório de Políticas Culturais (ObservaCult) & Brasil \\
\hline & Observatóriodos festivais & Brasil \\
\hline & $\begin{array}{l}\text { Observatório Missioneiro de Atividades Criativas e Culturais } \\
\text { (OMiCult) }\end{array}$ & Brasil \\
\hline & Observatório Cultural Unilasalle & Brasil \\
\hline & Observatorio Itaú Cultural & Brasil \\
\hline & Observatorio de Economía Creativa de Brasil (OBEC) & Brasil \\
\hline $\begin{array}{l}\text { Sistema Nacional de Informaciones e Indicadores Culturales } \\
\text { (SNIIC) }\end{array}$ & Brasil \\
\hline Observatorio de Diversidad Cultural & Brasil \\
\hline Observatorio de Políticas Culturales & Chile \\
\hline
\end{tabular}




\begin{tabular}{|c|c|}
\hline $\begin{array}{l}\text { Observatorio Iberoamericano de Cultura y Educación para la } \\
\text { Primera Infancia }\end{array}$ & Colombia \\
\hline Observatorio del Patrimonio Cultural y Arqueológico & Colombia \\
\hline Sistema Nacional de Información Cultural de Colombia (SINIC) & Colombia \\
\hline $\begin{array}{l}\text { Observatorio de Cultura Urbana del Instituto Distrital de Cultura y } \\
\text { Turismo }\end{array}$ & Colombia \\
\hline $\begin{array}{l}\text { Observatorio de Patrimonio Cultural MIA (Material, Inmaterial y } \\
\text { Arqueológico) }\end{array}$ & Colombia \\
\hline Observatorio del Caribe Colombiano & Colombia \\
\hline Observatorio Iberoamericano del Derecho de Autor (ODAI) & Colombia \\
\hline Observatorio de Comportamientos Culturales & Colombia \\
\hline Sistema de Información Cultural de Costa Rica & Costa Rica \\
\hline Observatorio Ecuatoriano de Gestión y Políticas Culturales & Ecuador \\
\hline $\begin{array}{l}\text { Observatorio Protección Internacional de la Cultura, del Patrimonio } \\
\text { cultural y de los Bienes culturales }\end{array}$ & España \\
\hline Observatorio ICOMOS del Patrimonio & España \\
\hline $\begin{array}{l}\text { Observatorio para la Investigación en Conservación del Patrimonio } \\
\text { Cultural }\end{array}$ & España \\
\hline $\begin{array}{l}\text { Observatorio del Patrimonio Cultural Subacuático Común y } \\
\text { Compartido }\end{array}$ & España \\
\hline Observatorio Extremeño de la Cultura & España \\
\hline Observatorio de la Cultura & España \\
\hline Observatorio de Cultura y Comunicación & España \\
\hline Observatorio da Cultura Galega & España \\
\hline Observatorio de los Públicos del Patrimonio Cultural de Cataluña & España \\
\hline Observatorio Navarro de la Cultura & España \\
\hline Observatorio de Igualdad de Género en el ámbito de la Cultura & España \\
\hline Observatorio Iberoamericano de Cultura & España \\
\hline $\begin{array}{l}\text { Fundación Interarts - Observatorio Europeo de Políticas Culturales } \\
\text { Regionales y Urbanas }\end{array}$ & España \\
\hline Kulturaren Euskal Behatokia (Observatorio Vasco de la Cultura) & España \\
\hline Observatorio Cultural del Proyecto Atalaya & España \\
\hline Monitors of Culture, Instituto de Estudios de Ocio & España \\
\hline Baròmetre de la comunicaciò i la cultura & España \\
\hline Observatorio de la Cultura de Zaragoza & España \\
\hline
\end{tabular}




\begin{tabular}{|c|c|}
\hline Observatorio de Industrias Culturales de Andalucía & España \\
\hline Sistema de información cultural de Guatemala & $\begin{array}{l}\text { Guatemal } \\
\text { a }\end{array}$ \\
\hline Observatorio de la Lectura & México \\
\hline Observatorio de Políticas Culturales de Veracruz & México \\
\hline Sistema de Información Cultural & México \\
\hline Observatorio Cultural de la Ciénega & México \\
\hline Observatorio de Politicas Culturales UACM & México \\
\hline Observatorio de Arte y Cultura & México \\
\hline Observatorio Cultural de Chiapas y el Sureste Mexicano (OCCHSM) & México \\
\hline Observatorio Económico de México & México \\
\hline Observatorio Frontera Sur de México & México \\
\hline Observatorio de Cultura Veracruz & México \\
\hline Observatorio Latinoamericano de Gestión Cultural & México \\
\hline Observatorio Cultural del Paraguay & Paraguay \\
\hline Observatorio de Políticas Culturales - Perú & Perú \\
\hline Observatorio Iberoamericano de Museos & Portugal \\
\hline $\begin{array}{l}\text { Observatório das Actividades Culturais (Observatorio de } \\
\text { Actividades Culturales) }\end{array}$ & Portugal \\
\hline Sistema de Información Cultural & Uruguay \\
\hline Observatorio Universitario de Políticas Culturales & Uruguay \\
\hline
\end{tabular}

Fuente: elaboración propia.

Las fuentes consultadas fueron los sitios web de los observatorios y otros documentos como notas periodísticas, artículos científicos, reportes técnicos, videos promocionales, etcétera. La estrategia de identificación de estas fuentes fue la utilización de buscadores, bases de datos, directorios y la implementación de la técnica de bola de nieve. La recuperación de los datos se llevó a cabo a través del llenado de un cuestionario con respuestas cerradas y abiertas, con un formulario virtual hospedado en la herramienta de Google Forms.

A partir de los datos recolectados, se realizó una categorización y codificación de las respuestas abiertas para identificar las coincidencias y las equivalencias. De esa manera, se definieron las generalizaciones que permitieron 
contabilizar las frecuencias estadísticas y las interpretaciones de tipo cualitativo. Los datos de las preguntas cerradas fueron la base del análisis estadístico descriptivo. Finalmente, la información documentada se registró en un formulario electrónico para la creación de fichas técnicas de cada uno de los observatorios, las cuales están disponibles para su consulta en el portal del Observatorio Latinoamericano de Gestión Cultural. ${ }^{8}$

La segunda fase de la investigación se realizará a partir de los resultados que aquí se presentan; para esto, se realizarán entrevistas a los responsables de los observatorios para recabar los datos faltantes, así como para ampliar y profundizar la información recolectada hasta el momento.

\section{Resultados}

A partir de la revisión de las fuentes consultadas, se recolectaron datos de la mayoría de los indicadores definidos en la metodología, con excepción de las líneas de investigación ${ }^{9}$, el año de creación y los alcances de la investigación. ${ }^{10}$ En ese sentido, al no contar con suficiente información actualizada, se optó por dejarlas fuera de este análisis y retomarlas para la segunda fase a través de las entrevistas.

\section{Ubicación geográfica}

Se identificó que la mayoría de los observatorios culturales se encuentran en España, pues representa $30 \%$ de la totalidad. El siguiente país con más registros es México con $17 \%$, seguido por Brasil con 16\%. Le siguen Colombia y Argentina con $12 \%$ y $9 \%$, respectivamente. Por su parte, Portugal y Uruguay solo tienen $3 \%$ cada uno (ver gráfica 1 ). Así, solo tres países concentran $63 \%$ de los observatorios culturales de Iberoamérica (ver gráfica 2).

\footnotetext{
8 Disponible en: https://observatoriocultural.udgvirtual.udg.mx/observatorios

${ }^{9}$ No se encontró información concreta sobre esta variable en la mayoría de los observatorios analizados. Si bien se podría intuir por los trabajos que publican o por los proyectos que desarrollan, se consideró que no había información pertinente para mostrarla como resultados, por lo que se tomará como elemento a observar en la segunda fase del proyecto.

10 Para las variables de año de creación y alcances de la investigación, se recolectaron datos de algunos observatorios, pero no era información suficiente (únicamente de una tercera parte) para realizar un análisis estadístico confiable. Por esto, también quedaron fuera de este análisis y se recabarán a través de las entrevistas que se realizarán más adelante.
} 


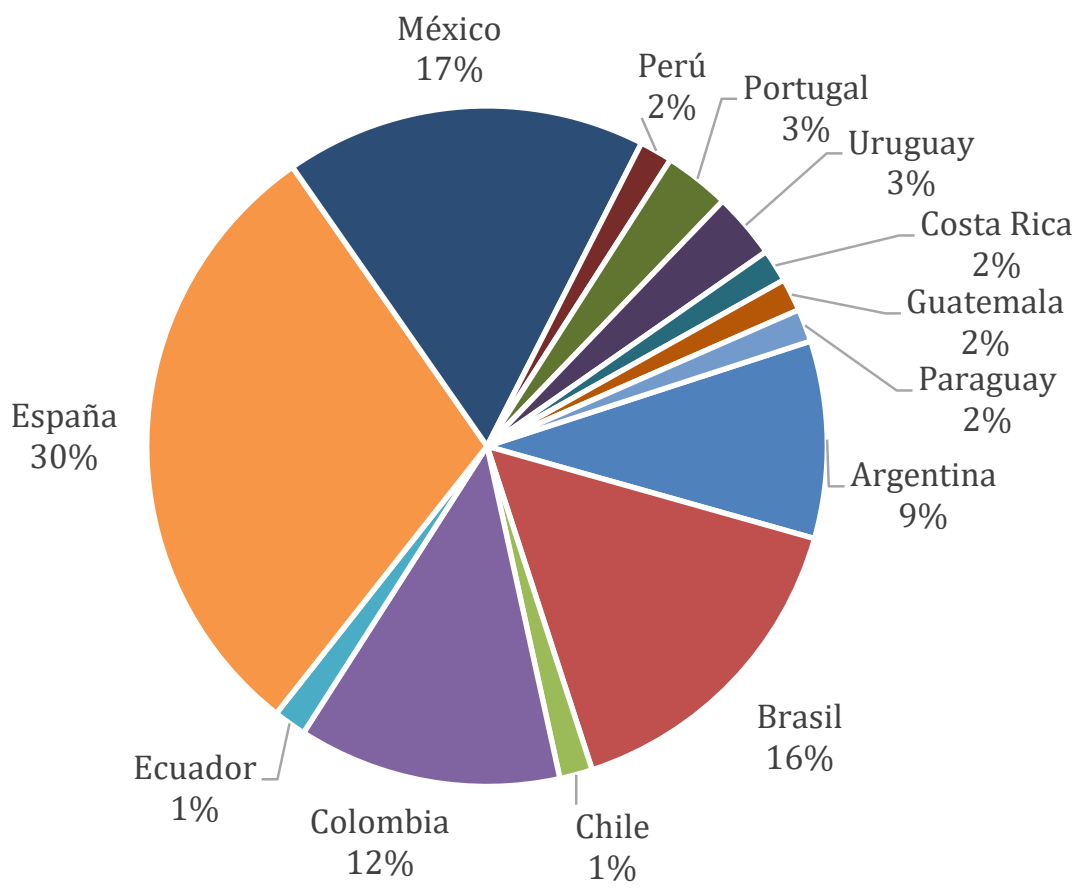

Gráfica 1. Ubicación de los observatorios por países.

Fuente: elaboración propia a partir de los datos empíricos.

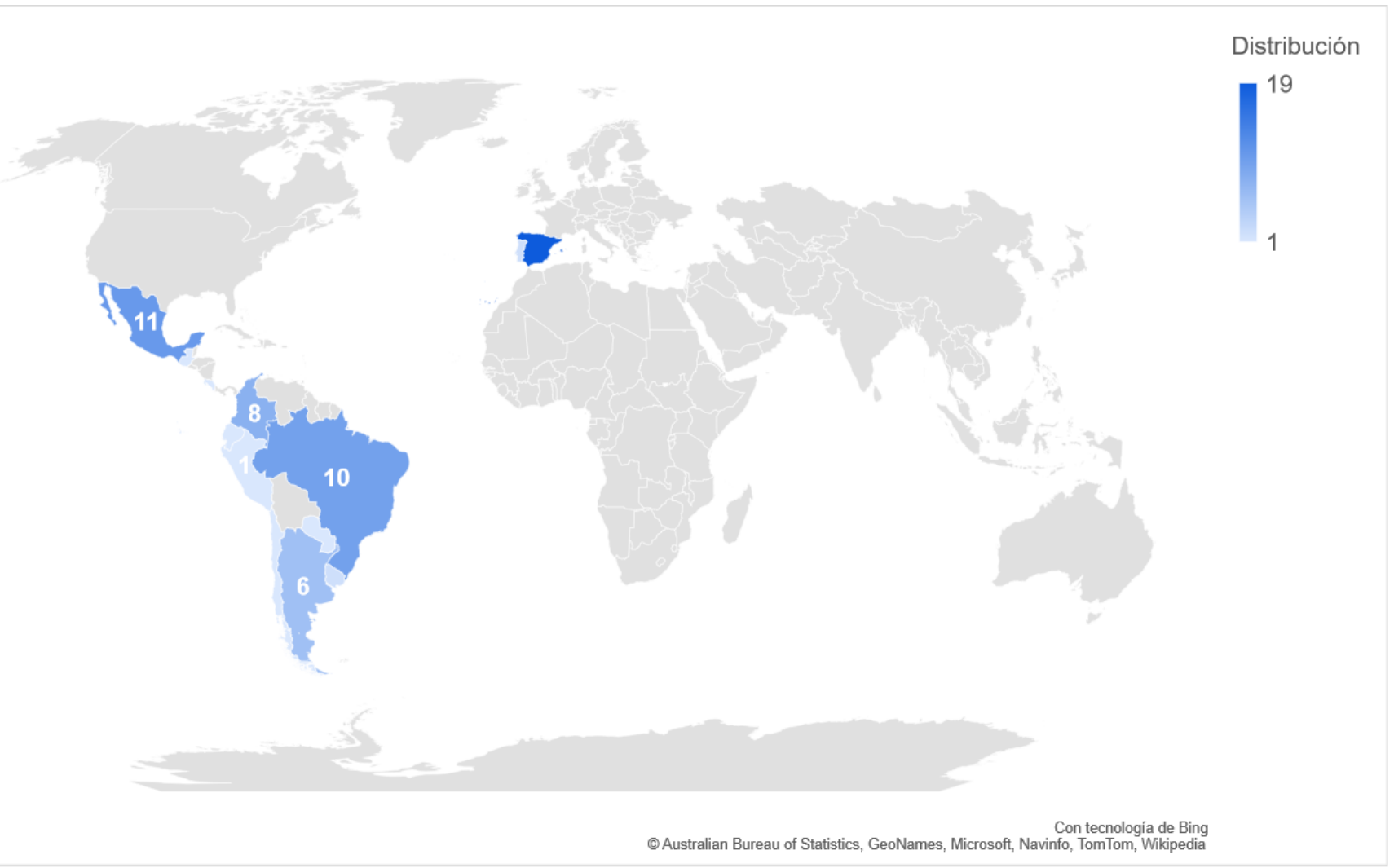

Gráfica 2. Distribución geográfica de los observatorios.

Fuente: elaboración propia a partir de los datos empíricos. 


\section{Temáticas de los observatorios}

La información recolectada muestra que la tendencia principal de las temáticas en los observatorios culturales son las políticas culturales, las actividades culturales y el patrimonio cultural (ver gráfica 3), las cuales suelen ser también frecuentes en las investigaciones de gestión cultural. Es necesario mencionar que se identificó que en los observatorios de creación más reciente prevalecían los de economía de la cultura, por lo que este tema seguramente será el más recurrente en los próximos años.

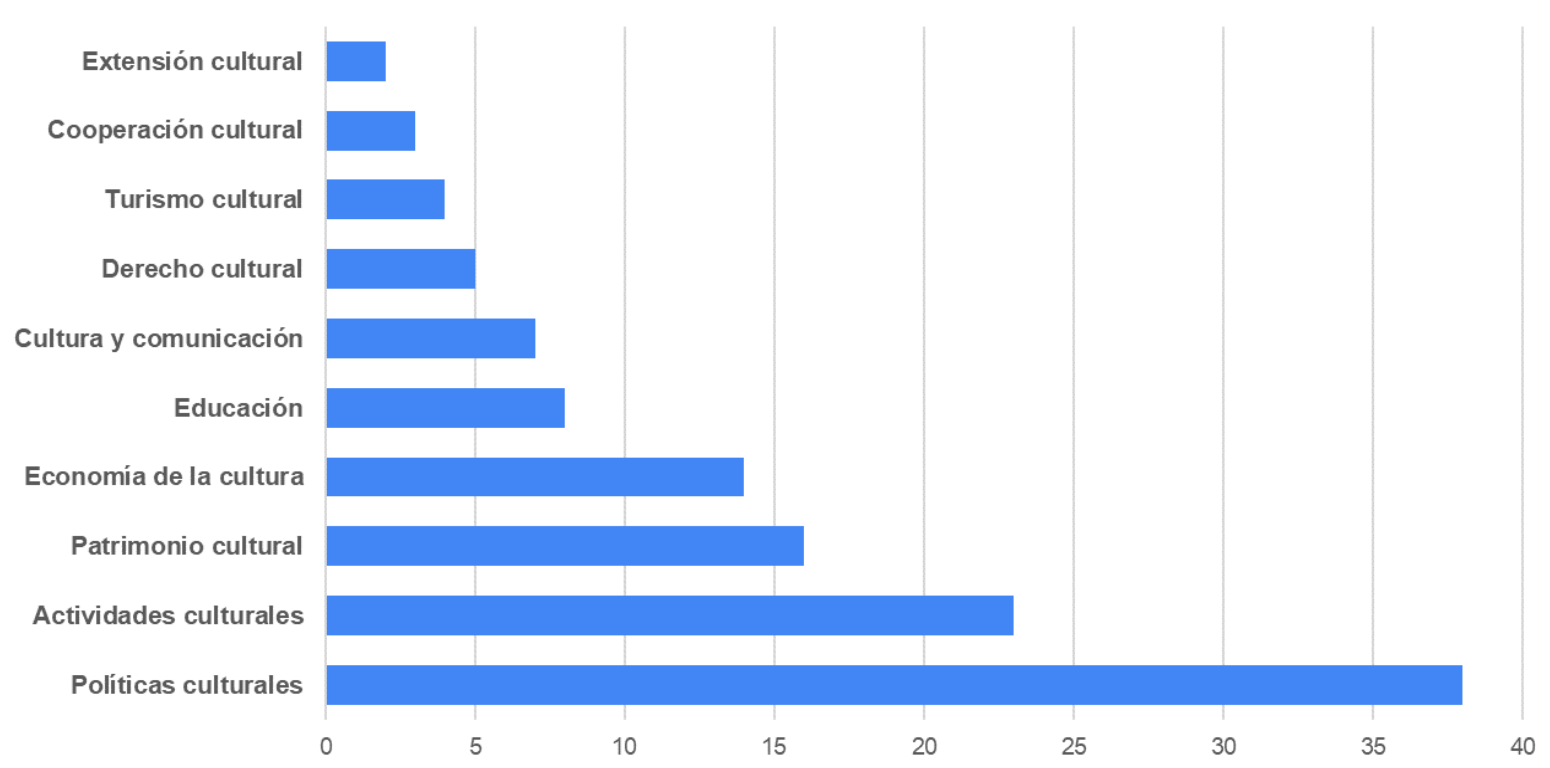

Gráfica 3. Frecuencias de las temáticas en los observatorios culturales. Fuente: elaboración propia a partir de los datos empíricos.

\section{Tipos de instituciones promotoras}

Los resultados muestran que las instituciones gubernamentales y las universitarias son las principales promotoras de los observatorios. Ambas representan $70 \%$ de la totalidad (ver gráfica 4 ). 


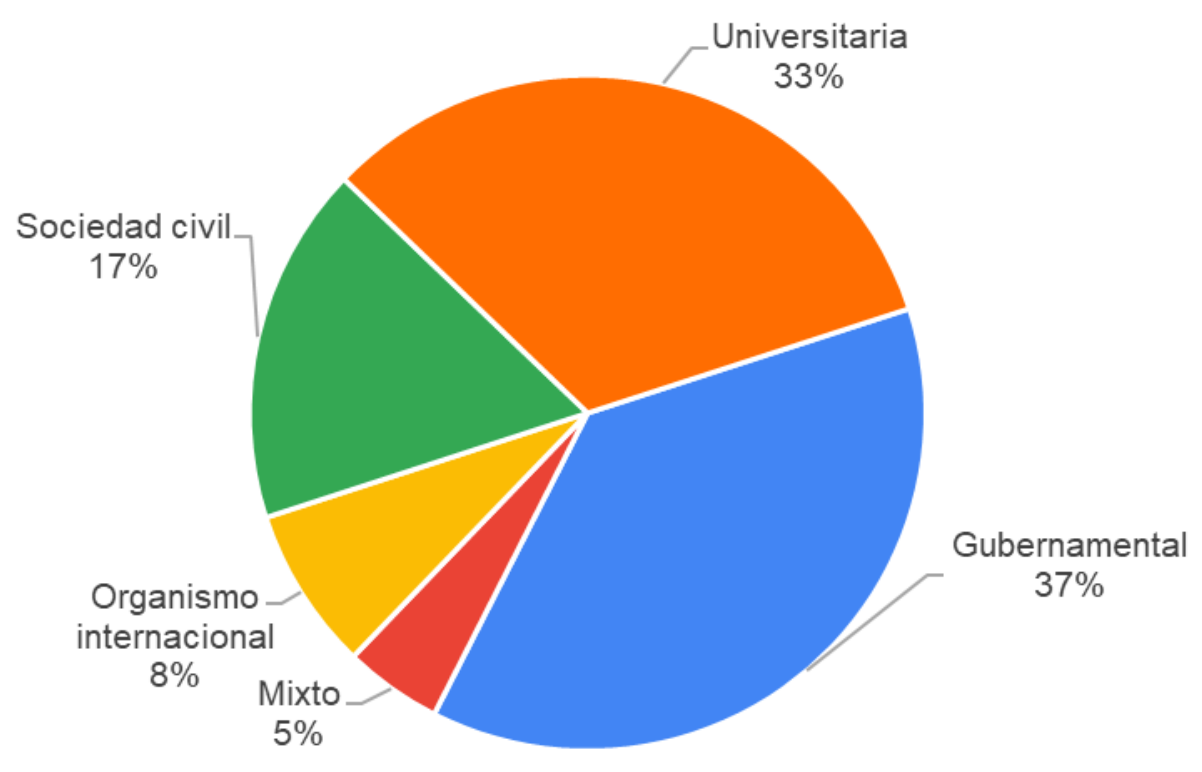

Gráfica 4. Tipo de institución promotora.

Fuente: elaboración propia a partir de los datos empíricos.

Al cruzar las variables de los tipos de institución con las temáticas, es evidente que las políticas culturales, las actividades culturales y la economía de la cultura predominan en las instituciones gubernamentales y universitarias. Sin embargo, la proporción de estas es muy alta en los observatorios gubernamentales comparados con los universitarios, que tienen una mayor variedad de temáticas (ver gráfica 5 ).

\section{Sobre sus modelos de trabajo}

En el análisis de las formas de trabajo utilizadas por los observatorios, se pudo identificar que los modelos más utilizados son los del sistema de información cultural (36\%) y los grupos de investigación (31\%). Solo se encontraron dos observatorios que tienen un modelo de gestión del conocimiento (ver gráfica 6). 


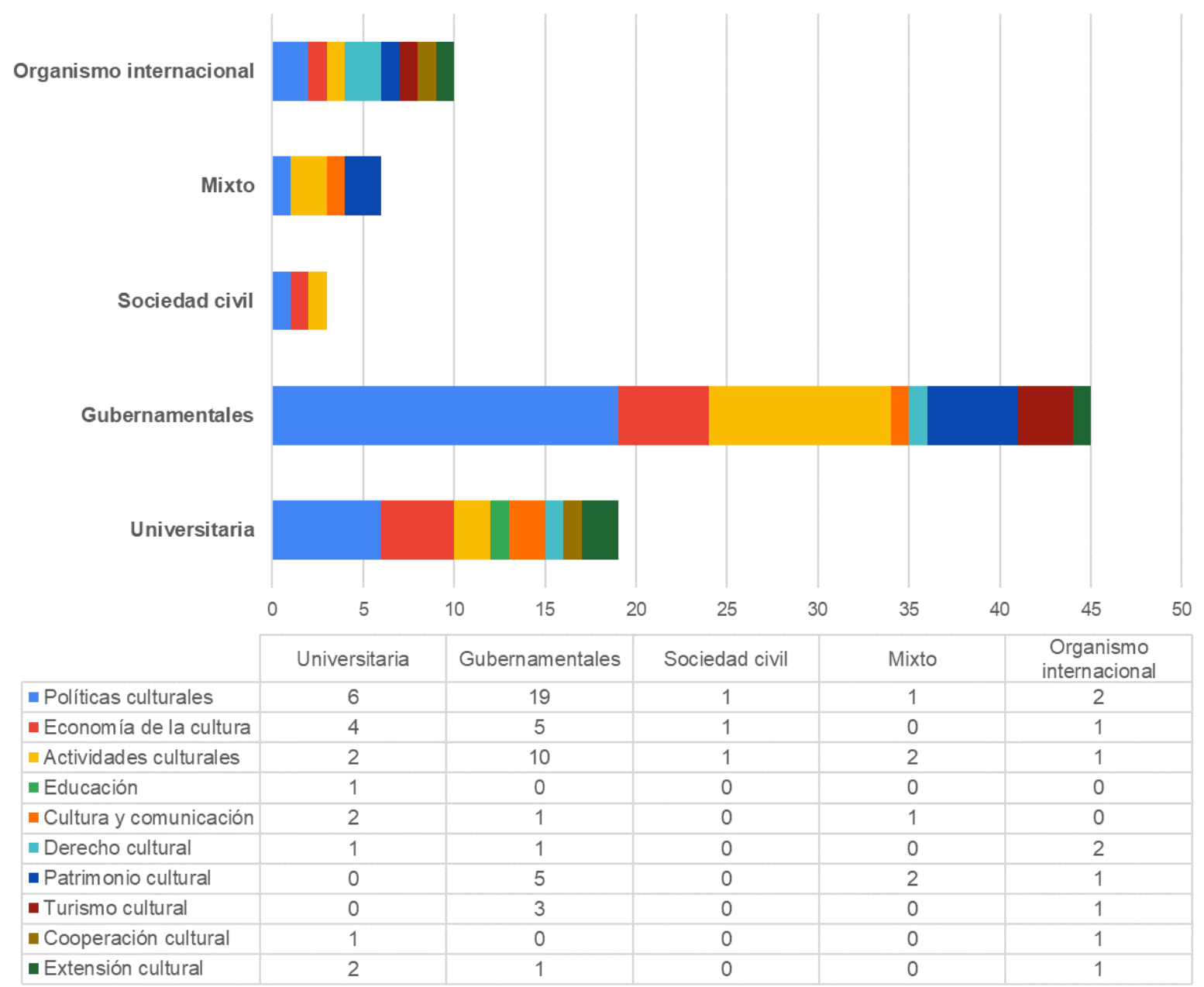

Gráfica 5. Frecuencias de las temáticas agrupadas por tipos de institución. Fuente: elaboración propia a partir de los datos empíricos.

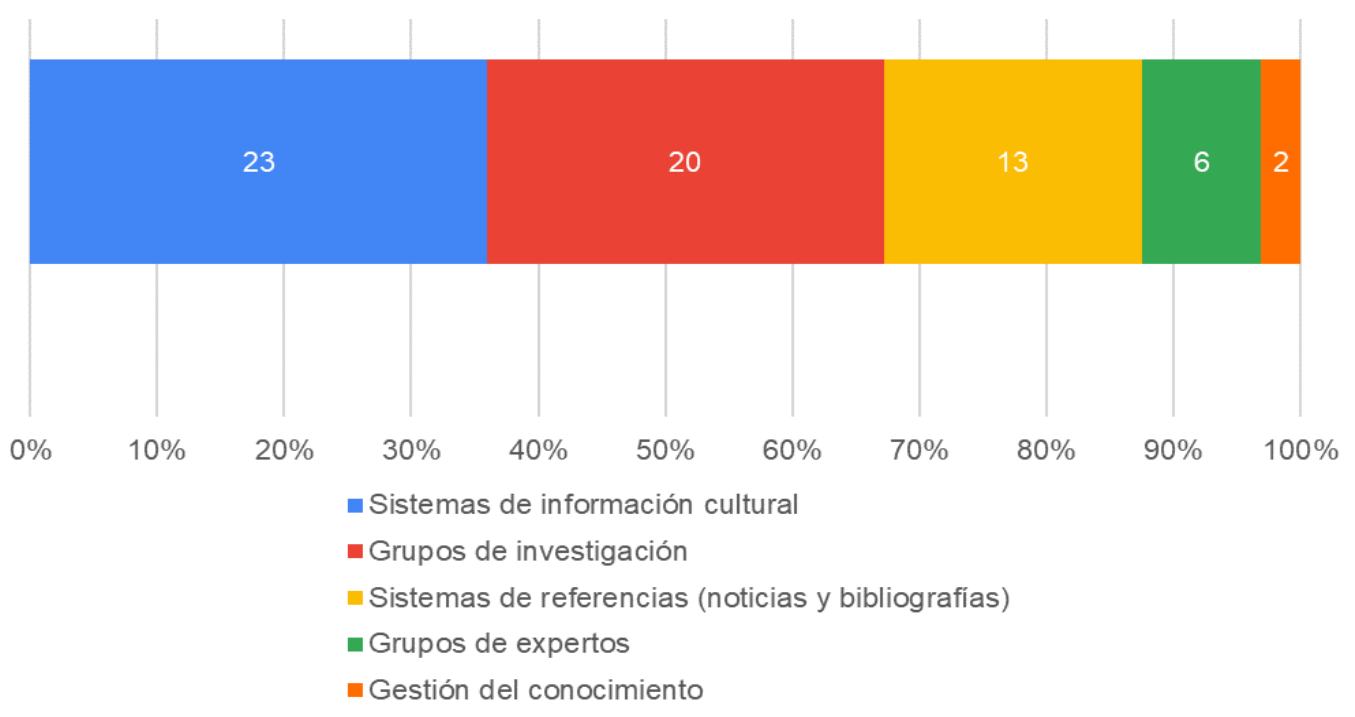

Gráfica 6. Modelos de trabajo de los observatorios.

Fuente: elaboración propia a partir de los datos empíricos. 
Cuando se agrupan estos modelos por el tipo de institución promotora, se observa que en los observatorios gubernamentales predomina el sistema de información cultural, mientras que en los universitarios prevalece el de grupos de investigación. En ese tenor, en las iniciativas de la sociedad civil predomina el sistema de referencias. Esto se explica porque las instancias gubernamentales suelen generar y conjuntar datos estadísticos a través de los censos. Por su parte, las universidades generan la información primordialmente por los proyectos de investigación, tanto de sus investigadores como de sus tesistas. La sociedad civil recaba y organiza la información que otras fuentes generan (ver gráfica 7).

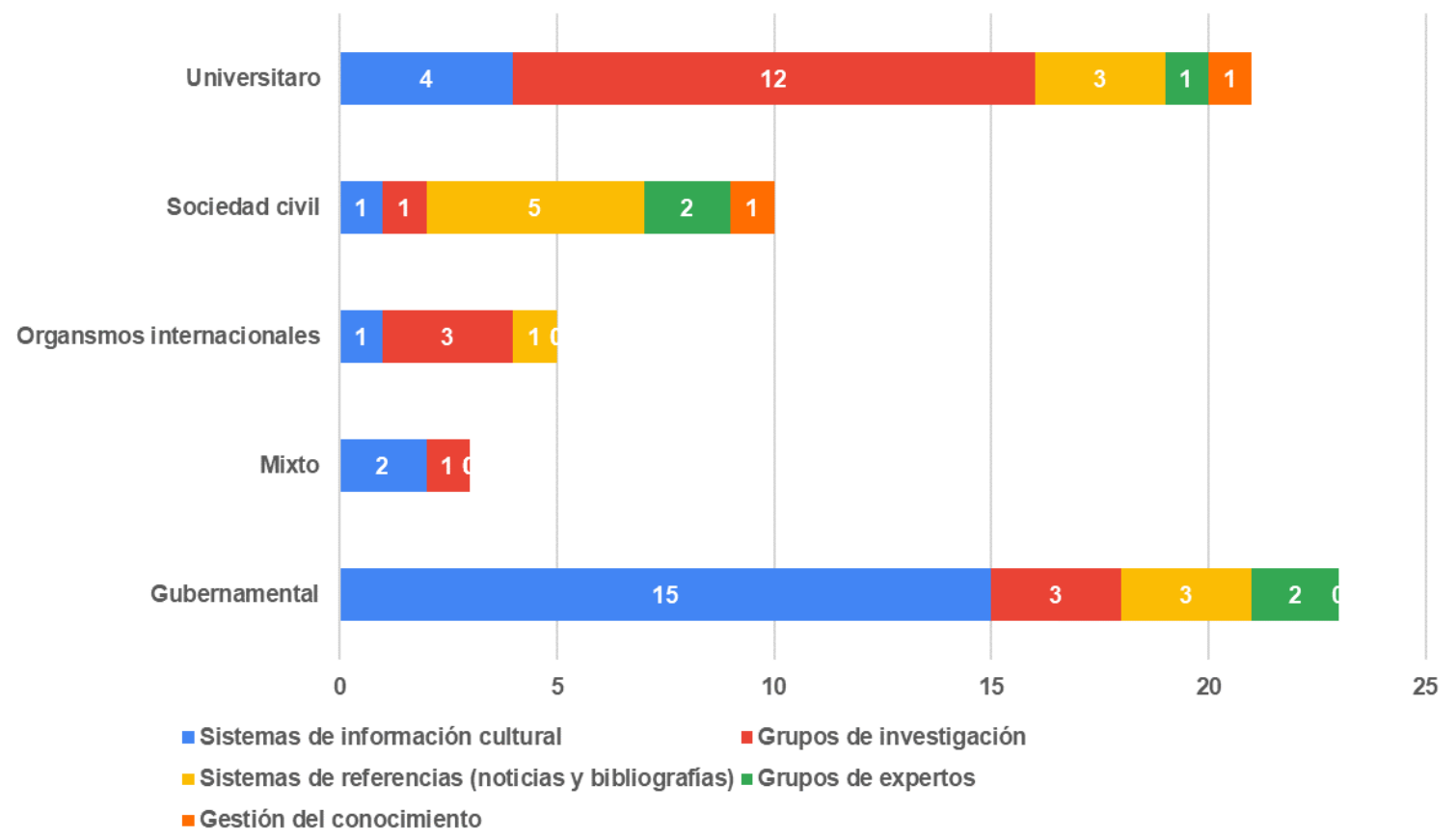

Gráfica 7. Modelos de trabajo por tipo de institución promotora. Fuente: elaboración propia a partir de los datos empíricos.

\section{Palabras clave}

Al tomar en cuenta el vocabulario controlado del Observatorio Latinoamericano de Gestión Cultural, se etiquetaron con palabras clave las investigaciones, las líneas de investigación y las publicaciones de los observatorios analizados. Este ejercicio brindó información relevante respecto a los temas de investigación relacionados con la gestión cultural que tienen más recurrencia. Así, se encontraron las políticas culturales, el patrimonio cultural, la administración cultural, el arte, los servicios culturales y la promoción y difusión cultural como las más recurrentes. Esta 
cuestión es comprensible, ya que estos son también los temas recurrentes en la profesión e investigación de la gestión cultural.

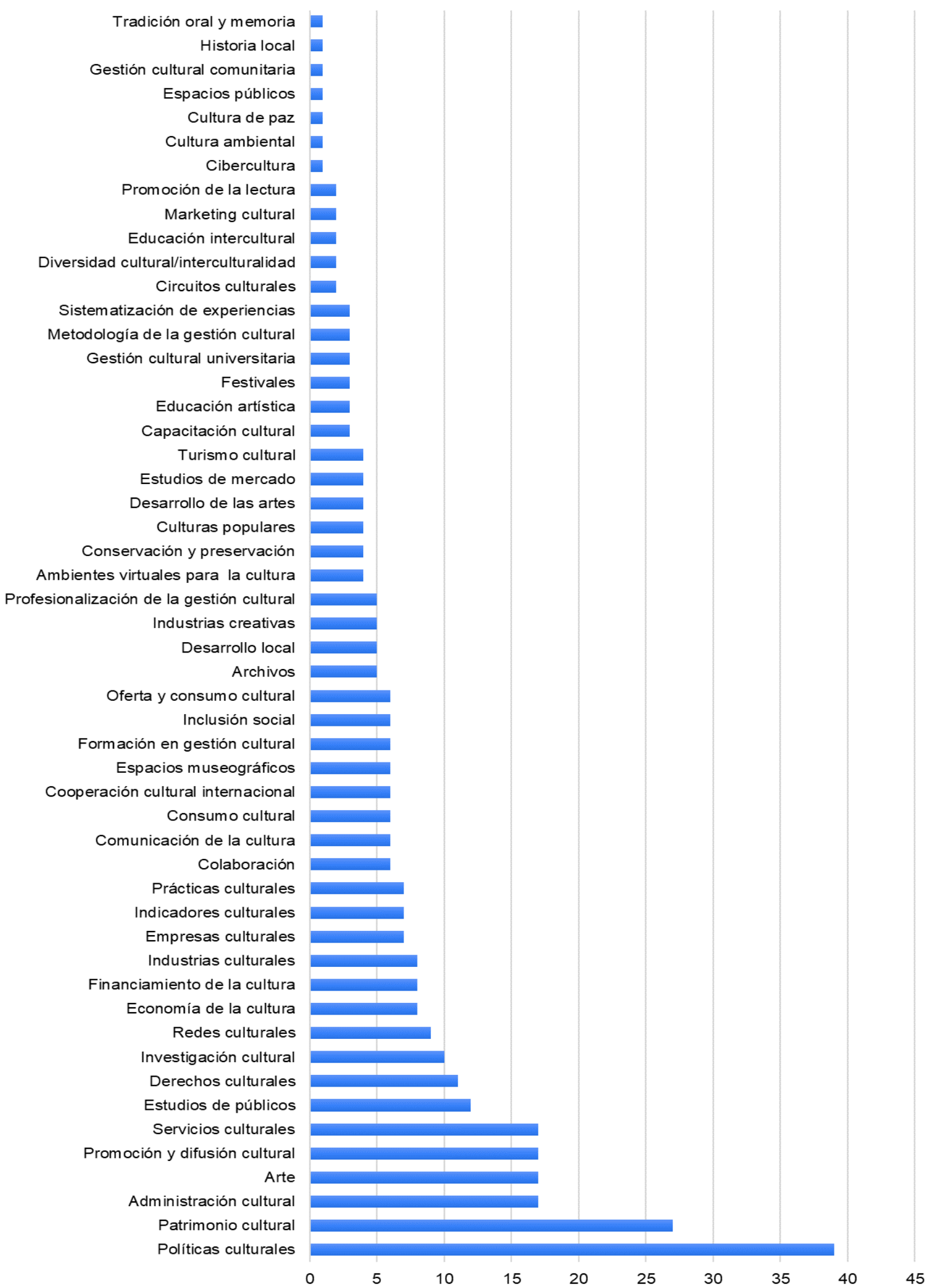

Gráfica 8. Frecuencia de las palabras clave en los contenidos de los observatorios. Fuente: elaboración propia a partir de los datos empíricos. 


\section{Objetivos y propósitos de los observatorios}

A partir de lo que cada observatorio analizado establece como sus objetivos o propósitos, se definieron las siguientes categorías analíticas y sus frecuencias (ver tabla 3).

Tabla 3. Objetivos o propósitos de los observatorios y sus frecuencias

\begin{tabular}{|l|c|c|}
\hline \multicolumn{1}{|c|}{ Objetivo o propósito } & Frecuencia & Porcentaje \\
\hline Diseñar y evaluar la acción cultural & 42 & 29.37 \\
\hline Inventario de bienes y servicios & 14 & 9.79 \\
\hline Generar bases de datos del sector cultural & 25 & 17.48 \\
\hline Realizar proyectos de investigación & 20 & 13.99 \\
\hline Análisis de los indicadores culturales & 12 & 8.39 \\
\hline Información para la toma de decisiones & 10 & 6.99 \\
\hline Socialización y transmisión del conocimiento & 18 & 12.59 \\
\hline Gestión del conocimiento & 2 & 1.40 \\
\hline
\end{tabular}

Fuente: elaboración propia a partir de los datos empíricos.

La mayoría de los observatorios tienen como fin diseñar y evaluar la acción cultural, así como generar bases de datos del sector cultural. Esto se debe a que el modelo de trabajo y la temática predominante son el sistema de información cultural y las políticas culturales, respectivamente.

\section{Actividades}

En su mayoría, los observatorios analizados tienden a difundir noticias del sector cultural, sobre todo aquellos que tienen un modelo de sistemas de referencias. Otras actividades recurrentes son las publicaciones, que suelen ser informes, diagnósticos y estados de la cuestión, pero también algunos libros y artículos en revistas indexadas. Menos de la mitad de los observatorios difunde noticias de actualidad relacionadas con el sector cultural y, en esa misma proporción, tienen 
repositorios de documentos, la mayoría de estos sin un sistema de gestión de colecciones digitales.

Así, se encuentra que la mayoría también realiza actividades de difusión y divulgación como congresos, seminarios, talleres, conferencias, que se suelen utilizar para dos fines: a) para la socialización de los resultados, y b) para recabar información de primera mano a través de la participación de expertos (modelo de grupo de expertos) y de los miembros del equipo (modelo de grupos de investigación), o bien, a través de una convocatoria abierta a los agentes culturales (ver gráfica 9).

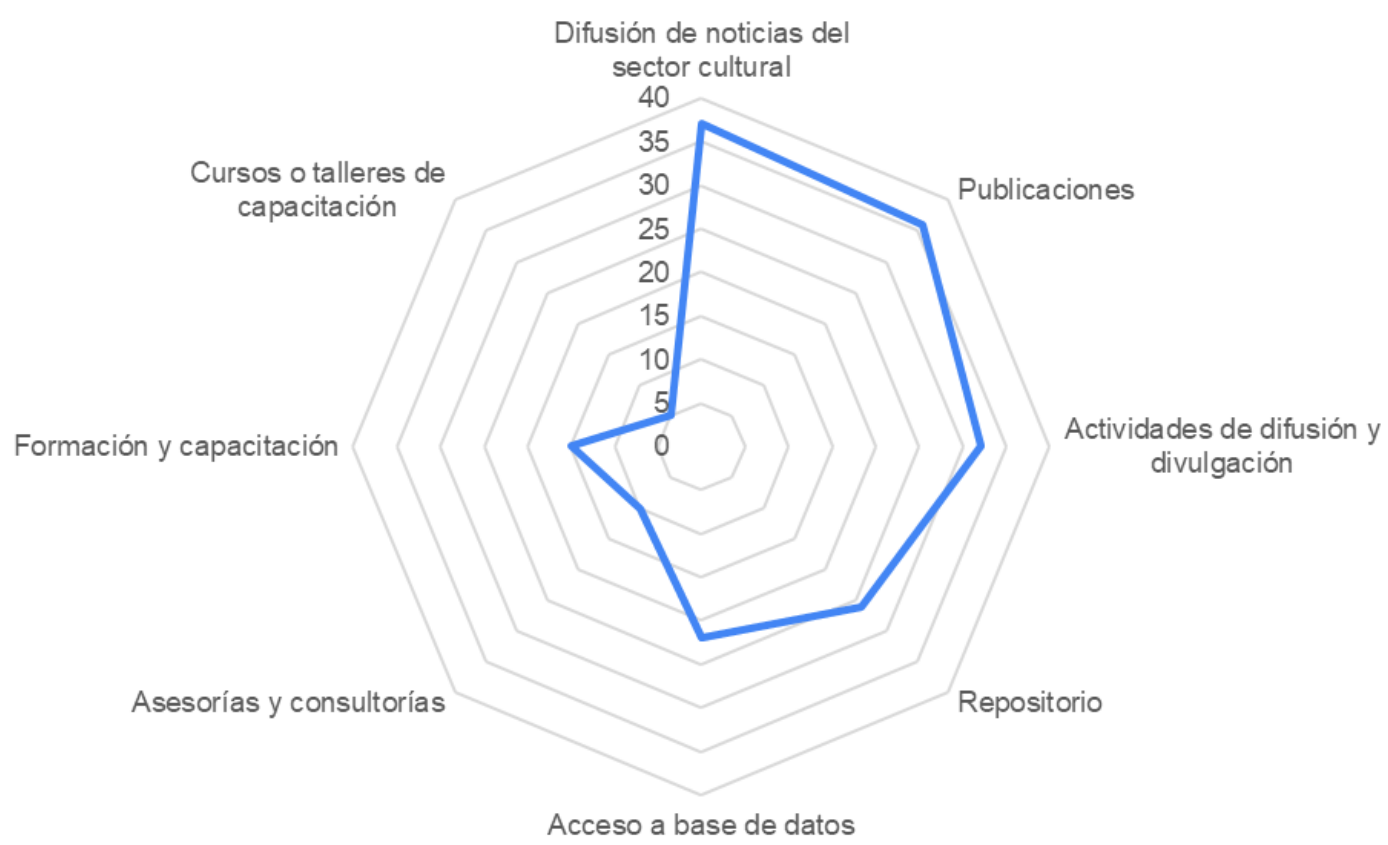

Gráfica 9. Frecuencia de las actividades para el logro de los objetivos. Fuente: elaboración propia a partir de los datos empíricos.

Otra cuestión relevante es la generación de repositorios, muchos de los cuales utilizan el mismo $\mathrm{CMS}^{11}$ como almacenamiento y organizador de los archivos; solo algunos utilizan programas de administración de colecciones digitales, ${ }^{12}$ como Dspace, Greenstone Digital Library, entre otros. Por su parte, el acceso a la base de datos es la actividad primordial (aunque no exclusiva) de los

\footnotetext{
11 Es el sistema de gestión de contenidos utilizado en los sitios web.

12 Este tipo de software permite etiquetar los archivos al generar y gestionar metadatos que pueden ser utilizados para las búsquedas, los procesos de interconexión con las bases de datos, el manejo de esos datos con fines estadísticos, entre otras funciones.
} 
observatorios con modelo de sistema de información cultural. Las actividades menos recurrentes son la formación, la capacitación, las asesorías y las consultorías.

\section{Dedicación a la gestión cultural}

La revisión de los datos recolectados mostró que la mayoría de los observatorios analizados realizan algunos trabajos ocasionales relacionados con la gestión cultural. En cambio, hay once observatorios que sí hacen explícito su interés y enfoque de observación desde la gestión cultural. Por otra parte, solo hay diez que cuentan con alguna línea de investigación relacionada con la gestión cultural (ver gráfica 10).

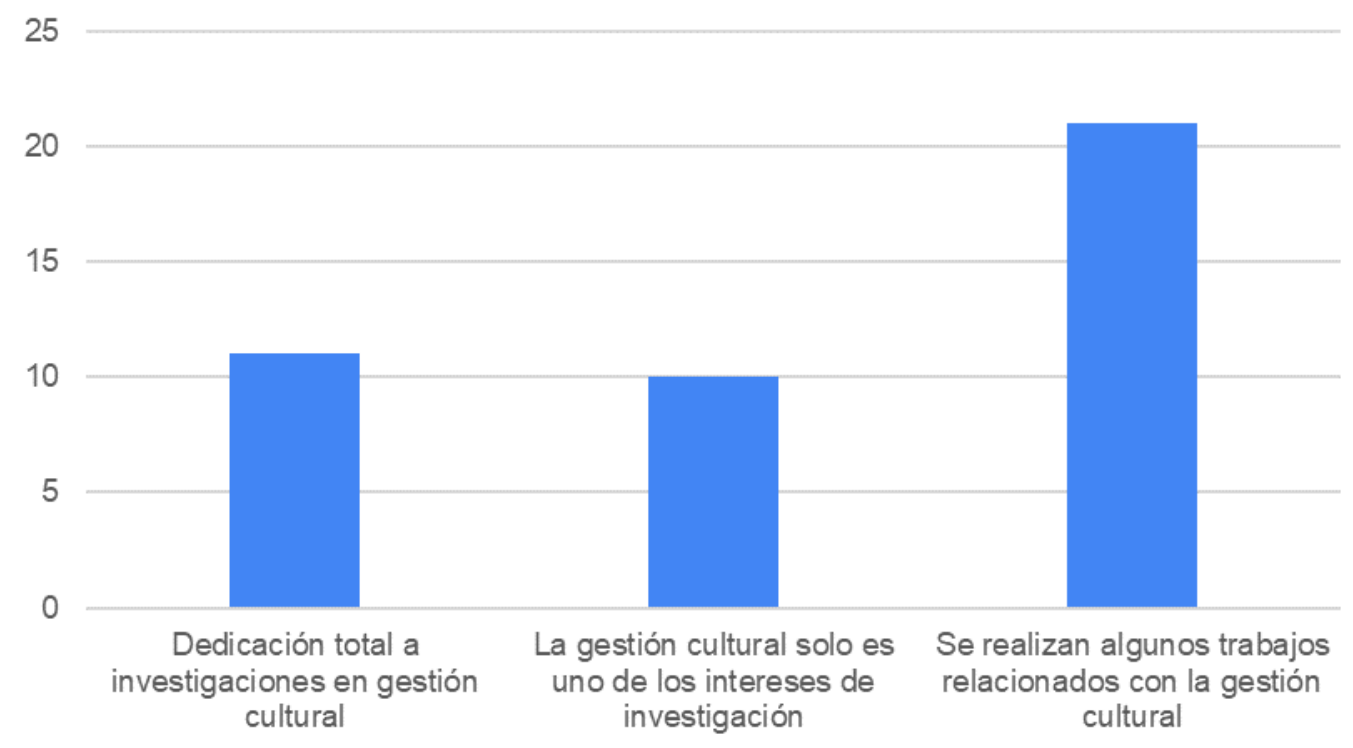

Gráfica 10. Dedicación a la gestión cultural de los observatorios culturales. Fuente: elaboración propia a partir de los datos empíricos.

\section{Conclusiones}

Hay un gran predominio de las políticas culturales y la economía de la cultura como temas de estudio en los observatorios culturales de Iberoamérica. Lo anterior puede deberse a que las instituciones gubernamentales promueven el surgimiento de observatorios de políticas públicas como una forma de orientar y legitimar las acciones gubernamentales, pero también porque las políticas 
culturales son uno de los temas de investigación con más trayectoria histórica en la gestión cultural.

En relación con los modelos de trabajo, predominan los de sistema de información cultural y los grupos de investigación, los cuales son promovidos por instancias gubernamentales para el primer caso, y por universidades para el segundo. Esto toma relevancia al observar los objetivos y las actividades para lograrlos, pues las instancias gubernamentales tienden a visualizar y operar los observatorios para diseñar y evaluar la acción cultural a través de la generación de bases de datos y la publicación de diagnósticos y resúmenes, con lo que esperan contar con la información para la toma de decisiones en la planeación y la administración cultural.

Por su parte, las universidades tienden a privilegiar la conformación de grupos de investigación que tengan como propósito generar y transferir nuevo conocimiento especializado a través del desarrollo de proyectos de investigación científica y humanística. La publicación de estos resultados, así como la sistematización y organización de la información documental en los repositorios institucionales, sirve como fuente de información en los procesos de formación y para nuevas investigaciones.

Otra tendencia evidente es que los observatorios cuyo modelo de trabajo es el sistema de referencias hacen énfasis en sus propósitos de conjuntar información (de fuentes secundarias) con el fin de comprender el sector y generar lineamientos para el diseño de la acción cultural. Para esto, difunden noticias del sector, realizan actividades de divulgación para darlo a conocer y, en algunos casos, elaboran publicaciones a manera de resúmenes.

En el estado del arte realizado se identificó que la tendencia actual de los observatorios sociales es trabajar en un modelo de gestión del conocimiento; sin embargo, de los casos analizados, solo dos tienen esta forma de trabajo. ${ }^{13} \mathrm{En}$ términos operativos, se encontró que un número significativo de sitios web de los observatorios no cuenta con información que permita conocer a detalle los proyectos que realizan, sus objetivos y líneas de investigación, pues centran más su atención en la difusión de las actividades de divulgación y en las publicaciones que realizan.

\footnotetext{
${ }^{13}$ El Observatorio ICOMOS de Patrimonio y el Observatorio Latinoamericano de Gestión Cultural.
} 
En la problematización y en el apartado teórico de este documento se mencionó la importancia de los observatorios como espacios de generación y transferencia de conocimiento especializado. Para el caso de la gestión y los observatorios culturales, esta función comienza a ser evidente, principalmente en tres aspectos:

1) La conformación y el acceso libre a las bases de datos culturales generadas a través de censos, encuestas, inventarios, conteos, etcétera, que suelen ser la materia prima para diversos diagnósticos, análisis y procesos de evaluación, ya sea de la acción cultural o de las problemáticas y necesidades en el sector cultural.

2) La conformación de grupos de investigación (especializados y multidisciplinarios) que desarrollen acciones investigativas y que, en muchos casos, estén ligadas a los procesos de formación en gestión cultural.

3) La publicación de libros, artículos, capítulos y demás producción académica, y su organización, catalogación y acceso libre a las personas interesadas en los temas que analizan con fines de intervención o investigación.

No obstante, y por la naturaleza de la metodología utilizada para esta fase de la investigación, aún quedan pendientes algunas preguntas por responder:

1) ¿Cuál es el impacto que han tenido los observatorios en el sector cultural? Sobre todo, en relación con los objetivos o propósitos que se plantean y las acciones que realizan para esto.

2) ¿Cuál es el grado de madurez institucional de los observatorios? Pues la información que se recolectó no es suficiente para conocer cómo se dan los procesos de formalización de estas instancias y, sobre todo, su permanencia en el tiempo.

3) ¿Cuál es la contribución de los observatorios en la conformación de una comunidad académica científica paradigmática que comparte lenguajes y marcos de referencias en el contexto del proceso de construcción de la gestión cultural como campo académico disciplinar? 
Estas son algunas preguntas que surgieron como parte de las conclusiones de la primera fase del mapeo de los observatorios culturales en Iberoamérica, las cuales podrían ser consideradas para avanzar hacia la comprensión y el entendimiento del estado actual de la formalización disciplinar e institucional de la gestión cultural.

\section{Referencias}

Abeledo, R. (2014). Un ejercicio de prospectiva en torno a los observatorios culturales. Periférica Internacional. Revista para el análisis de la cultura y el territorio, (14). 133-153. http://dx.doi.org/10.25267/Periferica.2013.i14.13

Angulo, N. (2009). ¿Qué son los observatorios y cuáles son sus funciones? Innovación Educativa, 9(47), 5-17. Recuperado de: https://www.redalyc.org/pdf/1794/179414895002.pdf

Arreola, V.; Brambila, B. y Mariscal, J. L. (2012). Profesionalización de gestores culturales en ambientes virtuales: el caso de la licenciatura en gestión cultural en línea. En J. L. Mariscal (ed.), Profesionalización de gestores culturales en Latinoamérica. Estado, universidades y asociaciones (pp. 85107). Guadalajara: Universidad de Guadalajara. Recuperado de: https://observatoriocultural.udgvirtual.udg.mx/repositorio/bitstream/han dle/123456789/87/Mariscal\%200rozco\%20-

$\% 20$ Profesionalizaci\%C3\%B3n\%20de\%20gestores\%20culturales\%20en \%20Latinoam\%C3\%A9rica.pdf

Ben, L. (2014). Utilidades de los observatorios culturales, la perspectiva práctica de los pararrayos. Periférica Internacional. Revista para el análisis de la cultura y el territorio, 79-89. http://dx.doi.org/10.25267/Periferica.2013.i14.10

Bayardo, R. (2018). Repensando la gestión cultural en Latinoamérica. En C. Yáñez (ed.), Praxis de la gestión cultural (pp. 17-31). Manizales: Universidad Nacional de Colombia. Recuperado de: https://observatoriocultural.udgvirtual.udg.mx/repositorio/bitstream/han dle/123456789/705/Praxis\%20de\%20la\%20gestio\%CC\%81n\%20cultura l.pdf

Brambila, B. A. (2015). Formación profesional de gestores culturales en México. Guadalajara: Universidad de Guadalajara. Recuperado de: http://biblioteca.udgvirtual.udg.mx/jspui/bitstream/123456789/1842/1/ FormacionPorfesional GestoresCulturales Brambila.pdf

Chevarria, F.; Inga, H.; De Pasquale, E. y Holmberg, K. (2015). Observatorios de políticas públicas en las Américas, una guía para su diseño e implementación en nuestras administraciones públicas. Washington: 
Organización de Estados Americanos. Recuperado de: https://www.oas.org/es/sap/dgpe/pub/ObservatoriosDePoliticasPublicas s.pdf

Giovinazzo, M. (2014). Observatorios culturales. Periférica Internacional. Revista para el análisis de la cultura y el territorio, (14). 69-77. Recuperado de: https://revistas.uca.es/index.php/periferica/article/view/2006

González, V. (2018). Avatares de la gestión cultural. Acercamientos desde la Licenciatura en Gestión y Promoción de las Artes de la UNICACH. Península, 13(2), 187-212. Recuperado de: http://www.scielo.org.mx/scielo.php?script=sci arttext\&pid=S187057662018000200187\&lng=es\&tIng=es

Instituto Nacional de Administración Pública, A. C. (2014). La función social de los Observatorios. El caso del Observatorio Latinoamericano de la Administración Pública (OLAP). Recuperado de: https://www.inap.mx/portal/images/pdf/book/praxis154.pdf

Mariscal, J. L. (2006). Formación y capacitación de los gestores culturales. Apertura, 6(4), 56-73. Recuperado de: https://www.redalyc.org/articulo.oa?id $=68800406$

Mariscal, J. L. y Rucker, U. (2016). Hacia la creación de sistemas de gestión del conocimiento en gestión cultural. El Observatorio Latinoamericano de Gestión Cultural. En J. Amaya, J. P. Rivas y M. I. Mercado (eds.). Diversidad, tradición e innovación en la gestión cultural. Teorías y contextos (pp. 73-91). México: Universidad de Guadalajara. Recuperado de:

https://observatoriocultural.udgvirtual.udg.mx/repositorio/bitstream/han dle/123456789/659/Diversidad-tradicion-innovacion-tomo1.pdf

Martinell, A. (2009). Las interacciones en la profesionalización en gestión cultural. Cuadernos del CLAEH, 32(98), 97-105. Recuperado de: https://publicaciones.claeh.edu.uy/index.php/cclaeh/article/view/13

Martínez, B.; Garrido, M. I. y Lugo, P. E. (2019). Observando observatorios. Un acercamiento a su conceptualización. Puebla: BUAP. Recuperado de: http://www.eco.buap.mx/publicaciones/libros/observando observatorios . pdf

Negrón, B. y Brodsky, J. (2014). 7.14 Los observatorios culturales hoy. En S. Catalán y A. J. González (eds.), Manual Atalaya Apoyo a la Gestión Cultural (1-12). Cádiz: Universidad de Cádiz y Observatorio Cultural del Proyecto Atalaya. Recuperado de: http://atalayagestioncultural.es/pdf/07.14.pdf

Rubim, A. A. C.; Barbalho, A. y Costa, L. (2012). Formação em organização da cultura: a situação latino-americana. Pragmatizes-Revista LatinoAmericana de Estudos em Cultura, 125-149. https://doi.org/10.22409/pragmatizes2.2.a10347 
Observatorio Cultural del Paraguay. (s. f.). Observatorio Cultural del Paraguay. Recuperado el 10 de marzo de 2021 de: http://www.observatoriocultural.gov.py/index.php

Observatório Cultural Unilasalle. (s. f.). Observatório Cultural Unilasalle. Recuperado el 15 de febrero de 2021 de: https://observatoriocultural.unilasalle.edu.br/

Observatório da cultura. (s. f.). Observatório da cultura. Recuperado el 28 de febrero de 2021 de: http://culturadesenvolvimentopoa.blogspot.com/

Observatorio da Cultura Galega. (s. f.). Observatorio da Cultura Galega. Recuperado el 31 de marzo de 2021 de: http://observatorio.consellodacultura.gal/

Observatorio de cultura y comunicación. (s. f.). Observatorio de cultura y comunicación. Recuperado el 18 de marzo de 2021 de: https://www.fundacionalternativas.org/cultura-y-comunicacion

Observatorio de Igualdad de Género en el ámbito de la Cultura. (s. f.). Observatorio de Igualdad de Género en el ámbito de la Cultura. Recuperado el 20 de febrero de 2021 de: http://www.culturaydeporte.gob.es/cultura/mc/espacio-deigualdad/observatorio-igualdad-genero-cultura.html

Observatorio de la Cultura. (s. f.). Observatorio de la Cultura. Recuperado el 15 de abril de 2021 de: https://fundacioncontemporanea.com/

Observatorio de la Lectura. (s. f.). Observatorio de la Lectura. Recuperado el 31 de enero de 2021 de: https://observatorio.librosmexico.mx/

Observatorio de los Públicos del Patrimonio Cultural de Cataluña. (s. f.). Observatorio de los Públicos del Patrimonio Cultural de Cataluña. Recuperado el 12 de enero de 2021 de: http://observatoripublics.icrpc.cat/

Observatório de Museus e Centros Culturais. (s. f.). Observatório de Museus e Centros Culturais. Recuperado el 1 de abril de 2021 de: http://www.fiocruz.br/omcc/cgi/cgilua.exe/sys/start.htm?tpl=home

Observatório de Políticas Culturais. (s. f.). Observatório de Políticas Culturais. Recuperado el 16 de marzo de 2021 de: http://observacult.org/

Observatorio del Patrimonio Cultural Subacuático Común y Compartido. (s. f.). Observatorio del Patrimonio Cultural Subacuático Común y Compartido. Recuperado el 2 de enero de 2021 de: https://www.observatoriogaleones.com/

Observatorio del patrimonio cultural y arqueológico. (s. f.). Observatorio del patrimonio cultural y arqueológico. Recuperado el 1 de febrero de 2021 de: https://opca.uniandes.edu.co/

Observatorio del Patrimonio Histórico Cultural. (s. f.). Observatorio del Patrimonio Histórico Cultural. Recuperado el 25 de febrero de 2021 de: 
https://www.buenosaires.gob.ar/cultura/patrimonio/valorizacion/observa torio-del-patrimonio-historico-cultural

Observatorio Extremeño de la Cultura. (s. f.). Observatorio Extremeño de la Cultura. Recuperado el 28 de enero de 2021 de: https://observaculturaextremadura.es/

Observatorio Iberoamericano de Cultura y Educación para la Primera Infancia. (s. f.). Observatorio Iberoamericano de Cultura y Educación para la Primera Infancia. Recuperado el 10 de abril de 2021 de: https://cerlalc.org/observatorio iberoamericano cultura educacion prim era infancia/

Observatorio Iberoamericano de Museos. (s. f.). Observatorio Iberoamericano de Museos. Recuperado el 5 de marzo de 2021 de: http://www.ibermuseos.org/acciones/observatorio-iberoamericano-demuseos/

Observatorio ICOMOS del Patrimonio. (s. f.). Observatorio ICOMOS del Patrimonio. Recuperado el 16 de enero de 2021 de: https://icomos.es/observatorio/

Observatório Missioneiro de Atividades Criativas e Culturais. (s. f.). Observatório Missioneiro de Atividades Criativas e Culturais. Recuperado el 25 de marzo de 2021 de: http://omicult.org/

Observatorio Navarro de la cultura. (s. f.). Observatorio Navarro de la cultura. Recuperado el 8 de abril de 2021 de: http://www.culturanavarra.es/

Observatorio para la Investigación en Conservación del Patrimonio Cultural. (s. f.). Observatorio para la Investigación en Conservación del Patrimonio Cultural. Recuperado el 25 de enero de 2021 de: http://www.investigacionenconservacion.es/

Observatorio Protección Internacional de la Cultura, del Patrimonio cultural y de los Bienes culturales. (s. f.). Observatorio Protección Internacional de la Cultura, del Patrimonio cultural y de los Bienes culturales. Recuperado el 12 de abril de 2021 de: https://portal.uc3m.es/portal/page/portal/inst fco vitoria/observatorios catedras/Observatorio\%20Protecci\%F3n\%20Internacional\%20de\%20la \%20Cultura\%20del\%20Patr

Observatório dos festivais. (s. f.). Observatório dos festivais. Recuperado el 3 de marzo de 2021 de: https://www.festivais.com.br/

Ortega, C. (2010). Observatorios culturales. Creación de mapas de infraestructuras y eventos. Barcelona: Ariel.

Ortega, C.; Claudio, M. y Bunten, A. (2011). Situación de los observatorios culturales en Europa. Informe SaCO. En C. Ortega (ed.), Nuevos desafíos de los observatorios culturales (pp. 135-166). Bilbao: Universidad de Deusto. Recuperado de: http://www.deustopublicaciones.es/ud/openaccess/ocio/pdfs ocio/ocio44.pdf 
Ortega, C. y Del Valle, R. (2010). Nuevos retos de los observatorios culturales. Boletín Gestión Cultural. No. 19. Observatorios culturales en el mundo. Portal Iberoamericano de Gestión Cultural.

Piedras, E. (2008). Indicadores de cultura. Observatorios culturales, ¿para qué? Este País, 35, 26-27. Recuperado de: http://archivo.estepais.com/inicio/historicos/209/26 cultura indicadores piedras.pdf

Puente, Y. (2016). Los observatorios sociales: plataformas para la socialización de la información y el conocimiento. Cuadernos de investigaciones de Ciencias de la Información, (1), 80-100. https://doi.org/10.34295/cuinci.vi1.8

Sistema de Información Cultural de Costa Rica. (s. f.). Sistema de información cultural de Costa Rica. Recuperado el 24 de abril de 2021 de: https://si.cultura.cr/

Sistema de Información Cultural de Guatemala. (s. f.). Sistema de información cultural de Guatemala. Recuperado el 20 de enero de 2021 de: https://www.sicultura.gt/

Sistema de Información Cultural. (s.f.). Sistema de Información Cultural. Recuperado el 28 de enero de 2021 de: https://www.gub.uy/ministerioeducacion-cultura/sic

Sancho, J. M. (2004). Los observatorios de la Sociedad de la Información: evaluación o política de promoción de las TIC en educación. Revista Iberoamericana de Educación, (36). Recuperado de: https://rieoei.org/historico/documentos/rie36a02.htm

Sarmiento R.; Delgado F. M. y Infante A. M. (2019). Observatorios: clasificación y concepción en el contexto iberoamericano. Revista Cubana de Información en Ciencias de la Salud (ACIMED), 30(2). Recuperado de: https://www.medigraphic.com/cgibin/new/resumen.cgi?IDARTICULO $=88939$

Vázquez, J. (2011). El observatorio de Políticas Culturales de la Universidad Autónoma de la Ciudad de México. Revista Digital de Gestión Cultural, 1(2), 79-81. Recuperado de: https://issuu.com/profesor.monzon/docs/rdgc numero 2

Zapata, C. M. (2004). Capacitación y formación. En B. Aceves (ed.), Primer Encuentro Nacional de Promotores y Gestores Culturales. Cuadernos de patrimonio cultural y turismo. No. 11 (pp. 131-138). México: Consejo Nacional para la Cultura y las Artes. 